\title{
Theoretical and Experimental of Evaluation of Flow Field Over a Double Delta Wing at Supersonic Speeds
}

\author{
Pavan Jacob Abraham, Ashish John Kalathil, Gopalsamy Murugesan, P. Name Jeyajothiraj
}

\begin{abstract}
Numerical and experimental investigations of flow over double delta wing at supersonic speed are investigated. For numerical simulations, the body geometry is generated using SolidWorks software, mesh is created using Gambit and solution is obtained using Fluent software. For the experimental studies, stainless steel model is fabricated and tested in the supersonic wind tunnel at Mach 2.5 at 0 and $5^{\circ}$ angle of attack. The results are presented in the present paper.
\end{abstract}

Keywords : Double delta wing, Supersonic Speed, Re-entry Launch Vehicle, Vortex shedding.

\section{INTRODUCTION}

A delta wing, is a triangle shaped wing, derived its name from Delta, the triangle-shaped letter in the Greek alphabet. In the case of the double delta wing, the strake is an additional surface which provides stable vortex over the delta wing. This improves the lift to drag ratio till the vortex break-down occurs [1]. The long root chord of the double delta wing can be built stronger, stiffer and at the same time lighter than a swept wing of equivalent lifting capability. It provides more internal volume for fuel and other storage without a significant increase in drag. Hence, the double delta wings are used in modern fighters and even in space transportation systems Space Shuttle and Re-entry Launch Vehicle (RLV).

S.B Kern [2] studied the effect of small surface modification (fillets) at the location of vortex shedding to control the shedding, its' path and vortex break-down. It was found that the lift increases by $14 \%$ at low angles of attack and by $18 \%$ at high angles of attack. N. G. Verhaagen et al [1] studied double delta wing flow field in low speed at angles of attack varying from -5 to $20 \mathrm{deg}$. both experimentally and numerically. Pressure sensitive paints are used to obtain vortex effect on surface of the wing. Results from the

numerical simulations compared with experimental values. O. Scott and L. William [3] investigated surface flow and

Revised Version Manuscript Received on 10, September 2019.

Mr. Pavan Jacob Abraham A, UG Scholar, Department of Aerospace Engineering, Karunya Institute of Technology and Sciences, Coimbatore, Tamilnadu, India.(Email: pavan.jacob22@gmail.com).

Mr. Pavan Jacob Abraham, UG Scholar, Department of Aerospace Engineering, Karunya Institute of Technology and Sciences, Coimbatore, Tamilnadu, India.(Email: ashishkalathi15@gmail.com).

Mr. Gopalsamy Murugesan, Research Scholar, Department of Aerospace Engineering, Karunya Institute of Technology and Sciences, Coimbatore, Tamilnadu, India.(Email: gsam19ni@ gmail.com).

Dr.P.Jeyajothiraj*, Professor, Department of Aerospace Engineering, Karunya Institute of Technology and Sciences, Coimbatore, Tamilnadu, India.(Email: jothirajpeter@karunya.edu) off-body flow at Reynolds numbers of 0.5 Million to 1.5 Million (based on the root chord). This study indicates roil up of the flow into a pair of primary vortices over the delta wing with axial velocity in the core of the vortex greater than twice free stream and with jet-like stream. Detailed review of studies carried out by NASA and Langley Research Center on vortex flow in supersonic flow is presented by Wood and Wilcox [4]. Missiles, wings and aircraft vortex flow studies in supersonic speeds from Mach number 1.5 to 4.6 are compiled and extensive analysis are carried out. Effects of vortex flow on vehicle performance are brought out. Interference between missile fins and body, impact of wing leading edge sweep, leading edge bluntness etc., are high-lighted in the review. Because of the importance of double delta wing in aerospace vehicles, a detailed theoretical and experimental investigations are carried out to analyze the flow field over a double delta wing at supersonic speed, $M$ $=2.5$. the results are presented in the present paper.

\section{METHODOLOGY}

\section{A. Model design}

The model chosen for the present study is a $76^{\circ}-40^{\circ}$ highly swept sharp edged double delta wing. The model is tested in the supersonic tunnel of Karunya Institute of Technology and Sciences. The test section size is $100 * 100 * 450 \mathrm{~mm}$. The model is designed in such a way that the blockage by the model is less than 5\% (for the range of angle of attacks tested) and dimensions are decided in a way to prevent the reflected shock from striking the model. The dimensions of the model thus obtained are shown in Figure 1. 


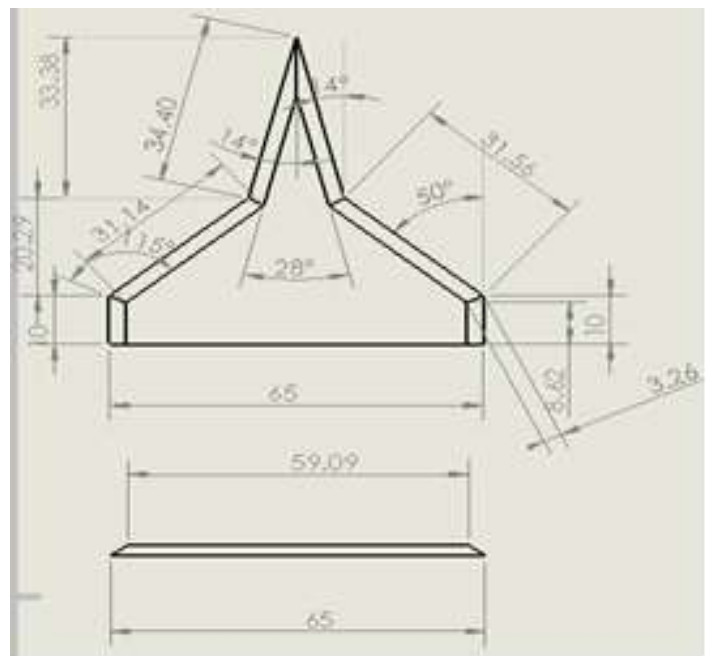

Fig.1: Configuration of the Double Delta wing.

The design of the model is carried out using Solid work. It provides a solid 3-D model and is shown in Figure 2. The total length and width of the model are $63.66 \mathrm{~mm}$ and $65 \mathrm{~mm}$.

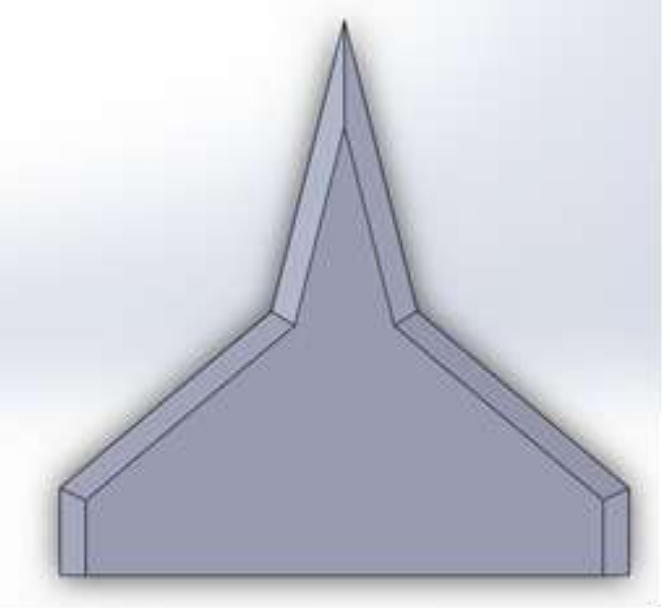

Fig.2: Three Dimensional model of the Double delta wing

\section{B. Model Mounting}

The stand part is made in solidwoks with the following dimensions. These dimensions mounting are obtained from a previously existing completed model. The inclined part of the mounting is kept at an angle of 50. The mounting dimensions and its 3D models are shown in Figure 3 and Figure 4 respectively. Fig.3: Configuration of the Mounting

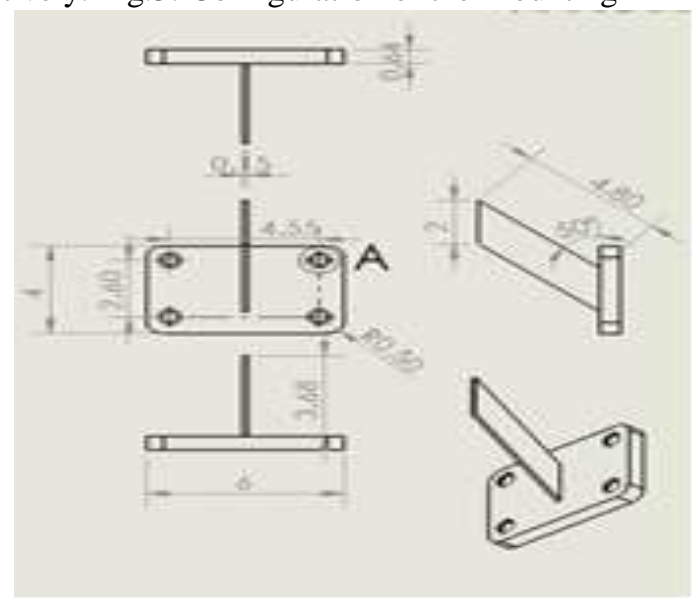

Fig.3: Configuration of the Mounting

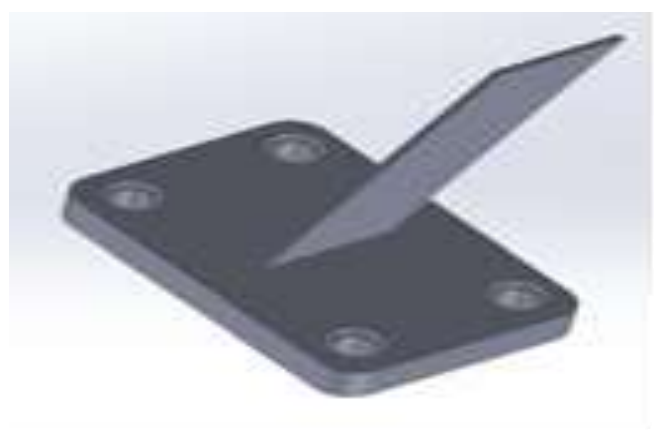

Fig.4:3D Model of the Mount

\section{Creating the body in the Design Modeler}

The model created in the Solidworks software is imported into ANSYS. After importing a model, the operation of the model must be changed from "Add Material" to "Add Frozen" so that the model will be in the solid form. After setting the geometry operations a domain is generated at a dimension similar to the test section of the supersonic wind tunnel. Length of the domain beind the model to the diffuser is around $45 \mathrm{~cm}$. An angle of attack can also be set for the swept double delta wing using the "rotate" option in the body transformation in the create bar. After selecting the option model must be selected for the rotate option to operate on, after the selection of the model the axis must be selected at which the rotation takes place. Only after settig the angle of attack can the domain be generated. The generated domain is shown in Figure 5.

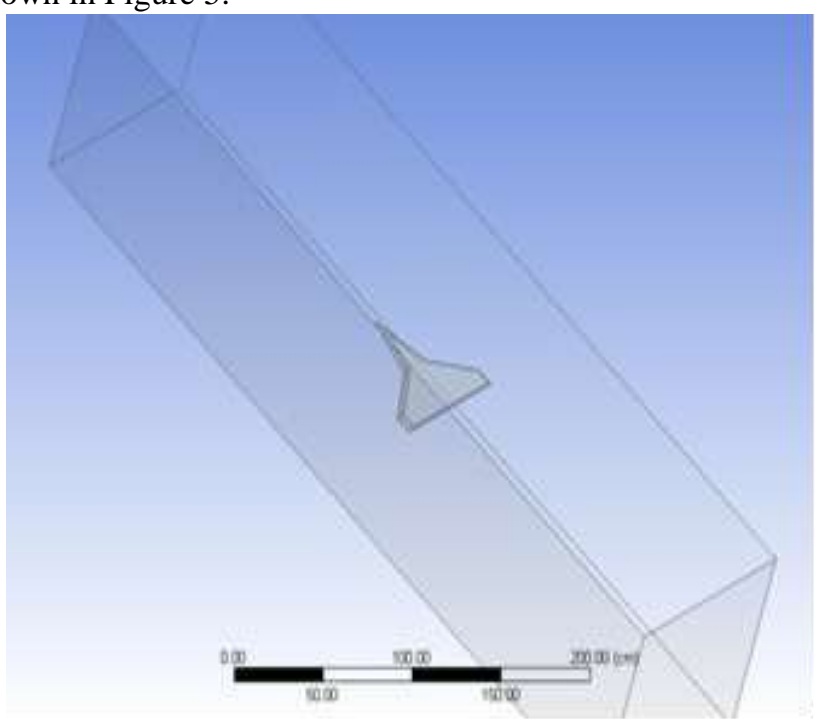

Fig.5: Domain for Numerical Simulation

\section{D.Generation of the Mesh File}

The "FLUID FLOW (FLUENT) ANSYS AUTODYN PREPOST" is the meshing software used and it is available for ANSYS v18.1. The model generated is meshed and then the sizing option is changed to make the mesh more fine, the size function is changed from curvature to adaptive and the relevance center is changed from coarse to medium. 


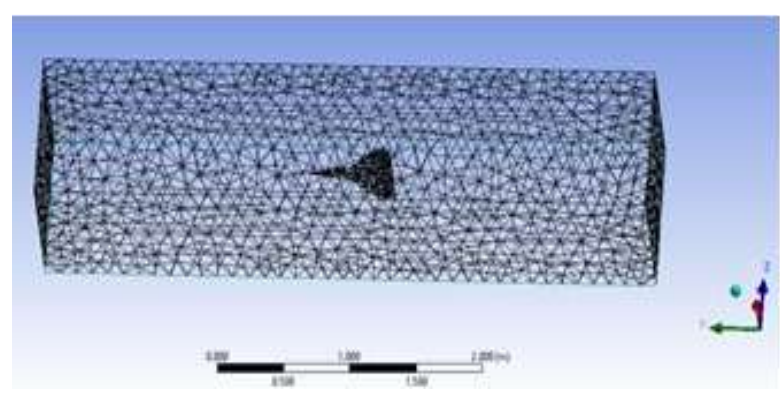

Fig.6: Generated Mesh over the model and the domain

After finishing the meshing the as shown in Figure 6, further meshing is done to make the model grid generation much more fine. To obtain a much finer mesh select the edges of the double delta wing where the mesh needs to be fine, select the edge option on the tool bar to select the desired edges. Right click and then go to insert and then select the edge sizing, this will help to set the desired sizing. This is done by changing the element sizing option to number of division's option in the definition, now the number of divisions for the edges is given as 40 . This will provide a really fine mesh at the edges. Then with the help of face mesh the top face of the double delta wing can also have a fine grid or the use of refine option can also provide a really fine mesh.

After obtaining the suitable mesh for the model the next step is to set the name selection for the inlet, outlet, wall and also for the double delta wing (DDW). This will help in identifying the face while providing the boundary condition so as to prevent any further confusions. The number of cells precent In the entire mesh is 273481 . The cell areas present are different due to the adaptive condition were given as the mesh condition

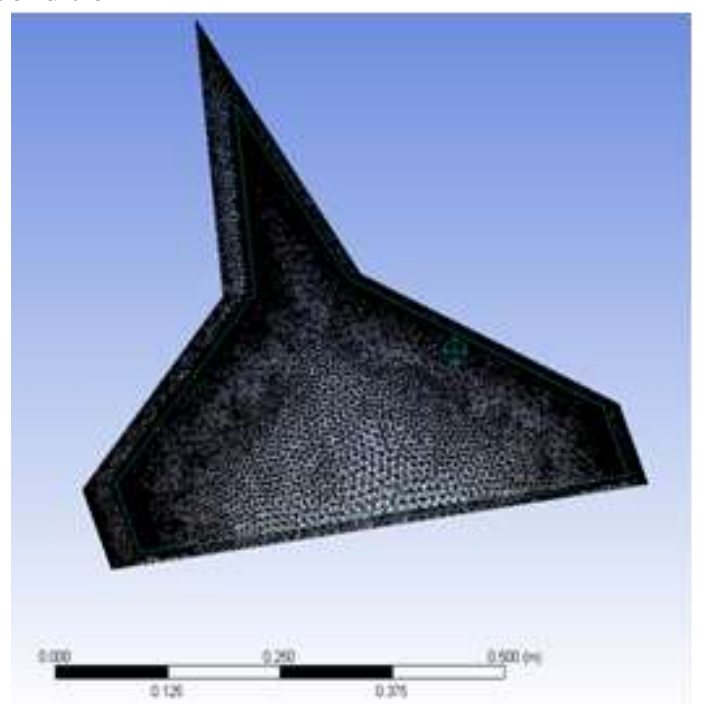

Fig.7: Mesh over the model

\section{SOLVER}

In the case of a solver, the entire model must be checked whether the mesh is fine whether the face area is accurate. Then the quality of the mesh is checked, the shape, whether it is structured or unstructured, checking the face area, checking the volume etc. In the case of quality check, it is used to check whether the grid that is generated is fine. The solver type will be density based because the flow over the model is a high speed compressible flow. The flow also is steady instead of transient. This is because steady state simulations, by definition are those whose characteristics do not change with time and whose steady conditions are assumed to be have reached after a relatively long time interval. After that the rest of the solution must be set, where the energy equation must be turned on which will allow you to set a parameter related to energy or heat transfer in the model. The Viscous Model dialog box allows you to set parameters for inviscid, laminar, and turbulent flow. The model that is used here is k-epsilon model, it is the most common model used in Computational Fluid Dynamics (CFD) to simulate mean flow characteristics for turbulent flow conditions. It is a two equation model that gives a general description of turbulence by means of two transport equations. The necessary boundary conditions needs to be in place for the inlet and outlet.

Boundary Conditions

Inlet- Velocity-Inlet

Outlet- Pressure outlet

Domain wall- Wall

The velocity inlet boundary conditions are used to define the flow velocity, along with all relevant scalar properties of the flow, at flow inlets. In this case, the total pressure or the stagnation pressure is not fixed but will rise to whatever value is necessary to provide the prescribed velocity distribution. This boundary condition is applicable to incompressible and compressible flows.

The pressure outlet boundary conditions require specification of a static (gauge) pressure at the outlet boundary. The relaxation factor provided is 0.25 . The solution methods provided for the flow, turbulence kinetic energy and turbulence dissipation factor are all given the second order upwind. Since the flow is implicit the Courent number for the this flow should be less than 1, the provided Courant number value is 0.5 and under the relaxation factor of 0.25 the turbulence Kinetic Energy, Kinetic dissipation rate and turbulent viscosity values are $0.8,0.8,1$.

\section{RESULTS AND DISCUSSION}

The turbulence model used is $\mathrm{K}$ - epsilon and solver is density based (compressible supersonic flow) and steady state solution is obtained. The mesh is imported and checked for the quality.

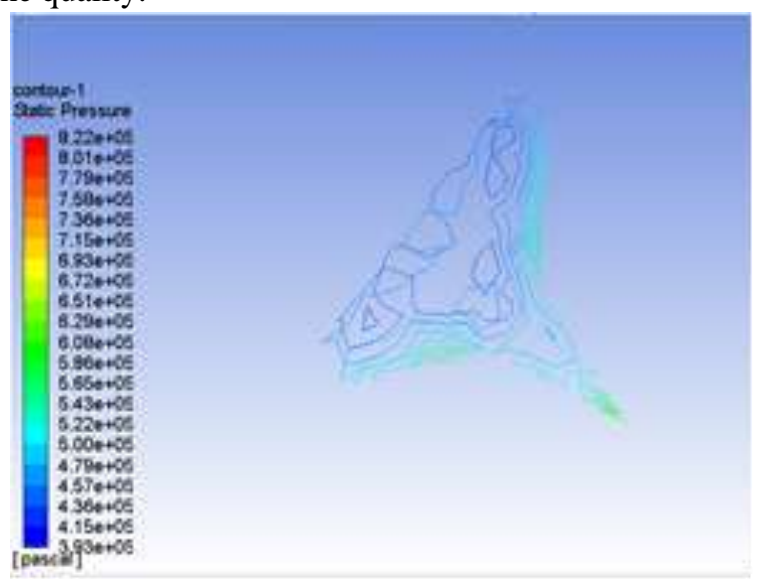

Fig. 8: Pressure contour at 15 angle of attack 


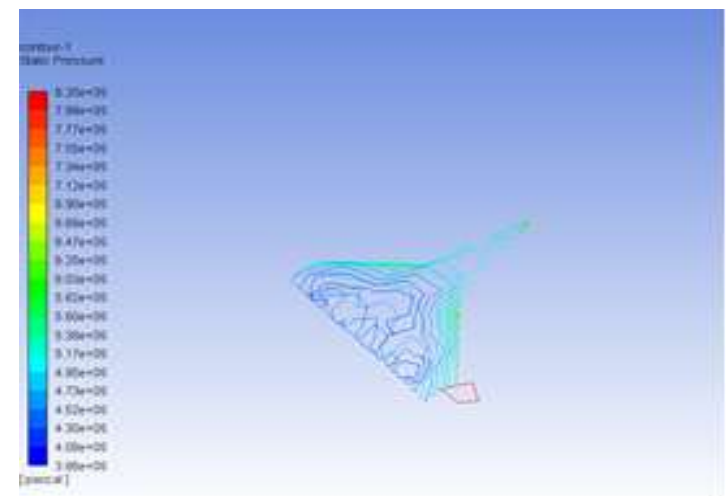

Fig. 9: Pressure contour at 0 angle of attack

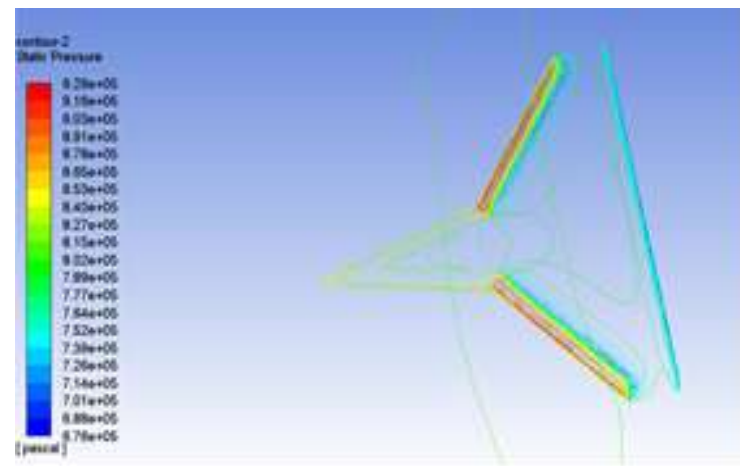

Fig. 10: Pressure contours at 5 ongle of attack

The pressure contours at 5 deg angle of attack id shown in Figure 10. High pressure levels are seen in the leading edge delta portion of the wing due to it $70 \mathrm{deg}$ inclination.

Pressure pallete for double delta wing at $55 \mathrm{deg}$. angle of attack and the pressure is higher near the center of the wing. Low pressure at the tip of delta portion is wing due to its high flow inclination and it is an indication of vortex formation.

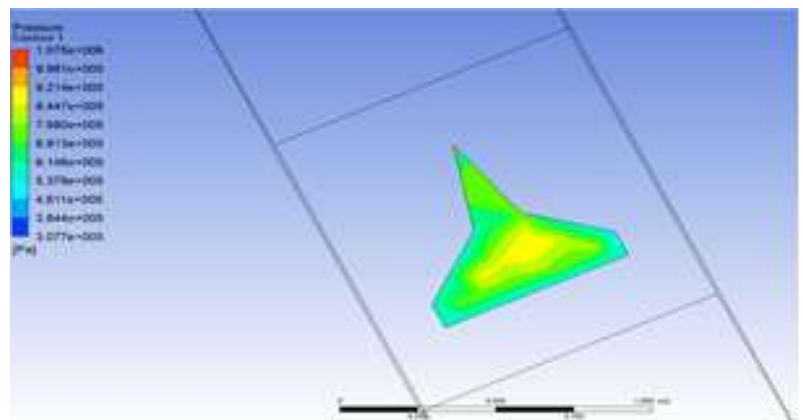

Fig. 11: Pressure distribution at 55 angle of attack

At 60 deg angle of attack (Figure 12), the pressure at the center of the wing is higher. This may be due to the reattachment/impingement of vortex on the wing.

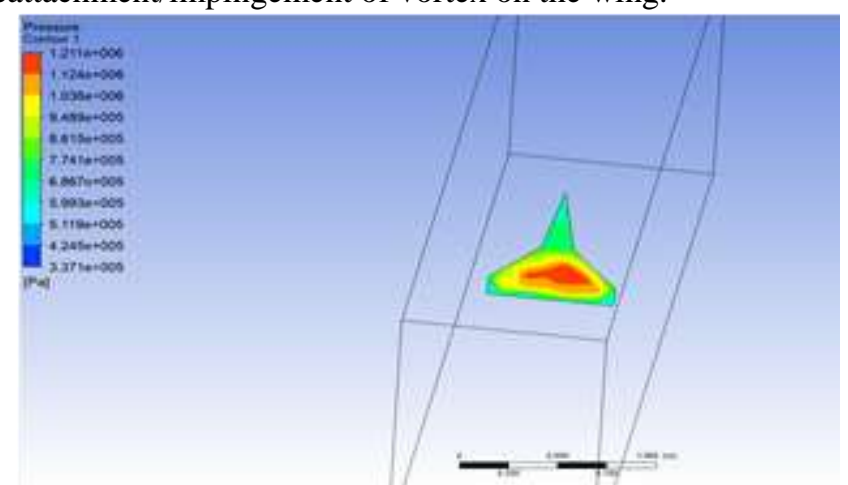

Fig.12: Pressure distribution at 60 angle of attack
Stream line pattern for angle of attack 60 and 65 deg are shown in Figure 13 and Figure 14 respectively. There figures indicate vortex formation and rolling up.

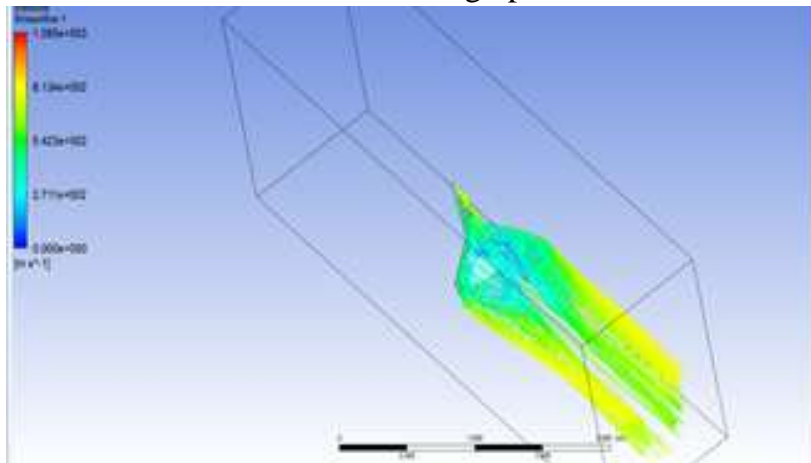

Fig.13: Velocity streamline at 60 angle of attack

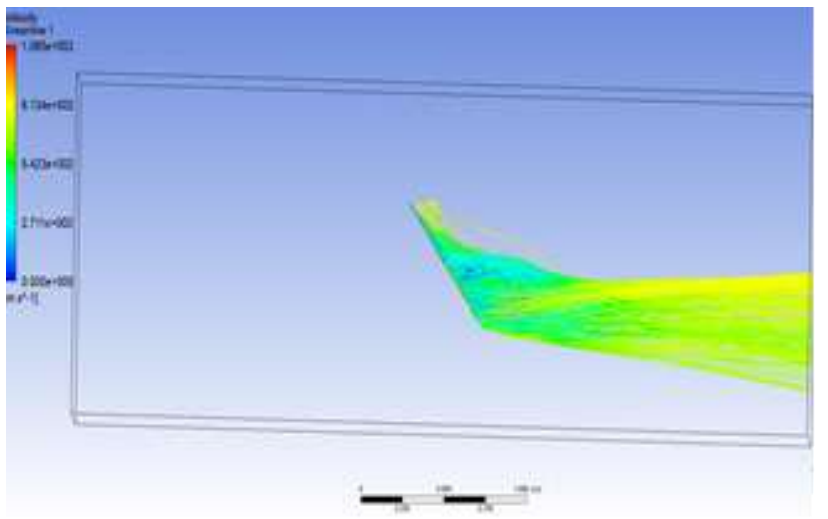

Fig.14: Velocity Streamline at 65 ongle of attack

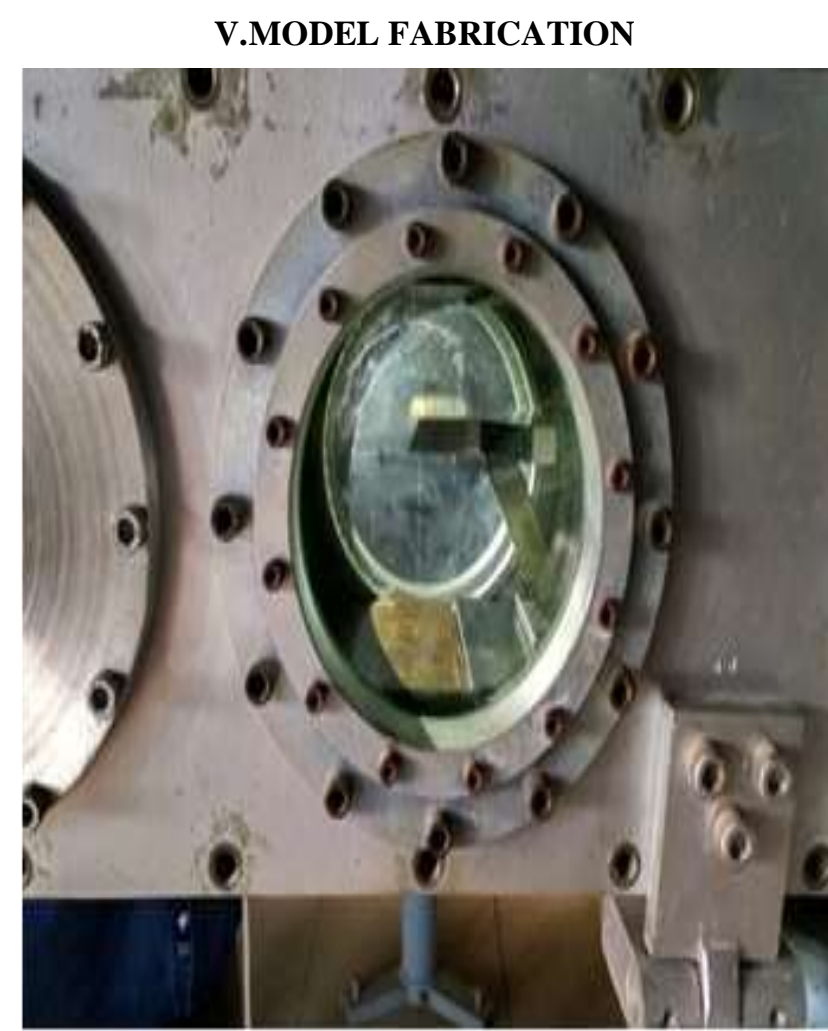

Fig.15: Fabricated model in Wind Tunnel

Published By: 


\section{EXPERIMENTAL ANALYSIS}

In the below figure 17 it shows flow over a double delta wing at Mach 2. The shock generated are bow shock at the stand and slight shock is generated at the tip of the model. The visualization technique used here is shadowgraph technique at Mach 2.

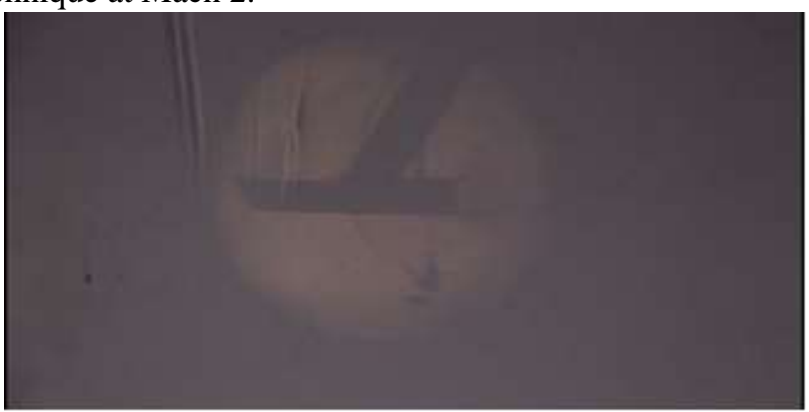

Fig.17: Flow over the double delta wing at 0 oangle of attack at Mach 2.5.

The shadowgraph technique is used here at Mach 2.5. The bow shock is generated at the inclined stand part and at the strake and at the wing a primary and secondary oblique shock is generated.

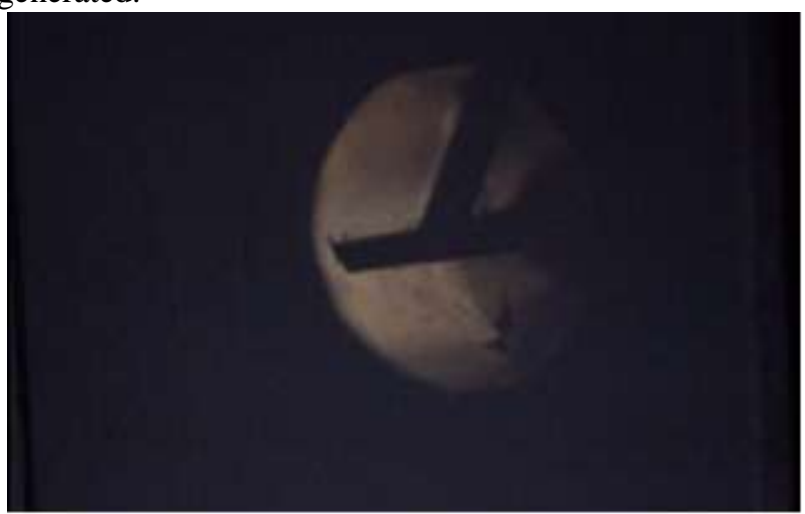

Fig.18: Flow over the double delta wing at 5 angle of attack at Mach 2.5

\section{CONCLUSION AND SCOPE}

The streamline flow over the double delta wing at different supersonic speeds is studied through numerical simulations. The pressure generated on the model increases with increase in the angle of attack, the pressure generation will be more concentrated on the top portion of the wing at $0^{\circ}$ angle of attack. As the angle of attack increases till $15^{\circ}$ the pressure is concentrated at the leading edge of the model. At higher angle of attacks the pressure will be more concentrated at the bottom of the wing. Using experimental analysis the shock generated over the double delta wing is visualized. The model is fabricated with high angle at edges and due to that the power of the shock will be more and the different types of shocks are generated on the model and the stand. An oblique shock is generated on the model and bow shock at the stand part.

\section{REFERENCES}

1. Steven B, Kern, "Vortex Flow Control Using Fillets on a Double-Delta Wing", Naval Air Warfare Center, Warminster, Pennsylvania 18974, JOURNAL OF AIRCRAFT, Vol. 30, No. 6, Nov.-Dec. 1993.

2. SukantaSaha, BireswarMajumdar, "Modeling and

Simulation on Double Delta Wing" International Journal of Advanced Computer Research (ISSN (print): 2249-7277 ISSN (online): 2277-7970) Volume-3 Number-1 Issue-8 March-2013, Research Fellow, Jadavpur University Professor, Jadavpur University jusukanta@gmail.com ssbmpub@gmail.com

3. N. G. Verhaagen, "A Study of the Vortex Flow over $76 /$ 40-Deg Double-Delta Wing", NASA Contractor Report 195032 ICASE Report No. 95-5.

4. Carine Viola Dsouza, "Numerical Simulation of 65 ? Delta Wing and 65 ?/40? Double Delta wing to study the behavior of primary vortices on aerodynamic characteristics" International Journal of Engineering Research and Technology, ISSN- 2278-0181, Vol. 4 Issue 06, June- 2015

5. Akira Oyama, Masato Ito et al, "Mach Number Effect on Flowfield over a Delta Wing in Supersonic Region", JAXA Institute of Space and Astronautical Science, Sagamihara, Kanagawa, 229-8510, Japan Article. January 2008 https://www.researchgate.net/publication/255457162.

6. H. J. STEWART, "THE LIFT OF A DELTA WING AT SUPERSONIC SPEEDS", California Institute of Technology, A. Busemann, Schriften der DeutschenAkademie fur Luftfahrtforschung, 7B, 105 (1943). Also Luftfahrtforschung, 12, 210 (1935), J Ackeret, Z.F.M., 16, 72 (1925), 5 H. Schlichting, Luftfahrtforschung, 13, 320 (1936)

7. Scott 0. Kjelgaard, William L. Sellers, "Detailed Flow-Field Measurements Over a $75^{\circ}$ Swept Delta Wing", Langley Research Center Hampton, Virginia, NASA technical

paper

2007,https://ntrs.nasa.gov/search.jsp?R=19910008717.

8. Richard M. Wood, Floyd J. Wilcox, Jr., e al, "Vortex Flows at Supersonic Speeds", NASA/TP-2003-211950, National Aeronautics and Space Administration Langley Research Center Hampton, Virginia 23681-219.

\section{AUTHORS PROFILE}
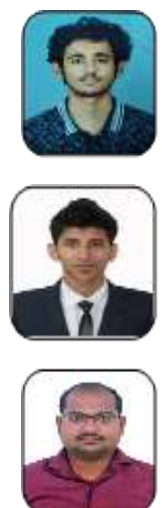

Gopalsamy Murugesan, Research Scholar, Department of Aerospace Engineering, Karunya Institute of Technology and Sciences, Coimbatore.

Dr. P. Jeyajothiraj, Professor, Department of Aerospace Engineering, Karunya Institute of Technology and Sciences, Coimbatore. Former Scientist 'G' from Indian Space Research Organization (ISRO) Specialization: Aerodynamics, Gas Dynamics, Experimental Aerodynamics, Acoustics and Aeroacoustics

Pavan Jacob Abraham, UG Scholar, B.Tech. Aerospace Engineering, Karunya Institute of Technology and Sciences, Coimbatore.

Ashish John Kalathil, UG Scholar, B.Tech Aerospace Engineering, Karunya Institute of Technology and Sciences, Coimbatore.

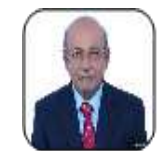

